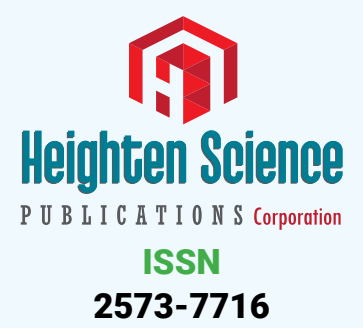

*Address for Correspondence: Eghoihunu Ireo, Scunthorpe General Hospital, Northern Lincolnshire and Goole NHS Foundation Trust, United Kingdom, Email: eghoihunu.ireo@nhs.net

Submitted: 09 August 2017

Approved: 21 August 2017

Published: 22 August 2017

Copyright: @ 2017 Ireo E, et al. This is an open access article distributed under the Creative Commons Attribution License, which permits unrestricted use, distribution, and reproduction in any medium, provided the original work is properly cited

Keywords: Lateral sinus thrombosis; Chronic suppurative otitis media, Mastoiditis
Case Report

\section{Otogenic lateral sinus Thrombosis: a rare complication of chronic Otitis media}

\author{
Eghoihunu Ireo*, Prashant Gupta and Ganapathy Dhanasekar \\ Scunthorpe General Hospital, Northern Lincolnshire and Goole NHS Foundation Trust, United \\ Kingdom
}

\section{Abstract}

Since the advent of antibiotics, lateral sinus thrombosis is an infrequent complication of otitis media. Lateral sinus thrombosis may occur by thrombophlebitis or penetration by offending pathogens through the dura of middle and posterior cranial fossae. We present a case of right-sided sigmoid and transverse venous sinus thrombosis as a rare complication of chronic suppurative otitis media in an adult. We discuss the patient's imaging, management and relevant literature to offer clinical recommendations.

A 39-year-old woman presented with headache, neck pain, vomiting, fever and photophobia with a tender right mastoid on examination. Computerised Tomography, Magnetic Resonance Imaging and Magnetic Resonance Venogram of the head revealed complete opacification of the right mastoid air cells and middle ear, with absent flow void in the right transverse and sigmoid sinus, consistent with thrombosis. After discussion with neurosurgery, she was commenced on anticoagulants. The patient was readmitted with right otalgia and otorrhea refractory to medical treatment, and ultimately underwent right mastoid exploration.

Conclusion: Lateral sinus thrombosis may occur with other intracranial or extracranial complications of otitis media. Clinicians should approach any complication of otitis media with vigilance as antibiotics may mask some signs and symptoms of mastoiditis, which can progress to otogenic brain abscess.

\section{Introduction}

Contemporary junior otorhinolaryngology trainees may be uncertain about diagnosing Lateral Sinus Thrombosis (LST) or unfamiliar with the management; as it is so infrequent they are unlikely to have participated in its management before [1,2]. We report a case of lateral sinus thrombosis and discuss investigations and management, summarised in an algorithm to guide readers.

The aetiology of LST involves mastoid bone erosion by cholesteatoma or granulation tissues causing the formation of a perisinus abscess [3]. Such tissue or abscess exerts pressure on the outer dural wall of the sinus and the intima, where fibrin, blood cells and platelets adhere forming a mural thrombus [3]. The resulting thrombus may become infected and propagate by thrombophlebitis via emissary vein, as well as by direct spread.

LST may accompany substantial morbidity and mortality [1,3]. It is now an unusual intracranial complication of otitis media, mastoiditis and cholesteatoma that accounts for $2-20 \%$ of intracranial complications of middle ear infections $[1,4]$. The contributing ear diseases have shifted over time from acute to chronic otitis media [4]. Generally intracranial complications of otitis media occur more readily in childhood [4] hence the rarity of this case. 
Lateral sinus thrombosis is characterised by pyrexia, with ear symptoms and signs such as otalgia, otorrhea, postauricular tenderness, and neurological symptoms and signs as headache, altered mental status and neck stiffness [1]. Nausea, vomiting, papilloedema, abducens nerve palsy, blurred vision and diplopia are reported from raised intracranial pressure, otherwise known as otitic hydrocephalus [1-3]. Gresinger's sign can be present (postauricular erythema and oedema) and To byAyer-Queckenstedt test may be positive-monitoring cerebrospinal fluid pressure (CSF) during a lumbar puncture, no increase of CSF pressure during compression of the internal jugular vein on the affected side, and an exaggerated response on the patent side, is suggestive of lateral sinus thrombosis [3].

LST was universally fatal until Lane performed the first successful operation for LST in 1888 [1-3]. Surgery initially reduced mortality to $50 \%$ [1,2], 40 years later, in 1928 the biologist Sir Alexander Fleming recognised the antibiotic substance penicillin, and stirred science to further improve the prognosis of LST. It was not until the early 1940 s that antibiotics were produced at large scales and employed to reduce mortality of otogenic LST to 5\% [1]. Historically the hallmark of otogenic LST was a triad of spiking fever (caused by escape of beta-haemolytic streptococcus in the circulation), headache and middle ear disease. The spiking fever is scarcely observed today, the use of antibiotics is reported to mask it [3,4].

Over the last few decades, imaging has evolved from roentgenograms to detailed, non-invasive imaging such as Computer Tomography (CT) with contrast and Magnetic Resonance Venography (MRV), and the mortality rate has continued to decline to less than $1 \%$ as management becomes more sophisticated.

\section{Case Report}

A39-year-old lady presented to the Emergency Department with a two-week history of worsening headache, associated with fever, vomiting, neck pain and photophobia. She had a one-year history of recurrent ear infections, which started during her second pregnancy, but no previous surgery to either ears or the head. On examination there was right post-aural tenderness and positive neck stiffness. There was no otorrhea, or rash and she scored 15/15 on the Glasgow Coma Scale. The patient was investigated extensively to find the cause of her symptoms.

CT head revealed that the right mastoid air cells and right middle ear to be opacified. MRI Head showed lateral sinus thrombosis. Moreover, MR Venogram confirmed thrombosis of the right transverse and sigmoid sinuses that was the same length as the sinuses and showed altered signal on T1 images. The primary diagnosis was right lateral sinus thrombosis.

Treated with analgesia and broad spectrum antibiotics for mastoiditis and anticoagulants were started, aiming for a target INR 2-3. She was discharged once her symptoms settled with advice to seek medical attention if she has any bleeding as she is on therapeutic low molecular weight heparin (LMWH).

One month later she was re-admitted to hospital with right-sided otalgia and headache. CT Mastoids (Figure 1) again showed complete opacification of the right mastoid air cells and middle ear cavity with soft tissue density material. Right middle ear ossicles were not identified. As there was no previous surgical intervention then the appearances was considered to be due to complete erosion of the middle ear ossicles by the soft tissue density within the middle ear cavity (Figures 2,3).

MRI Head (Figures 4,5) also showed right middle ear opacification with absent flow void in the right transverse and sigmoid sinus consistent with thrombosis. C-reactive protein was 138, and International Ratio INR of 1.1. She was started on Meropenam 2 grams IV, TDS and Metronidazole 500 mg IV TDS, and Gentisone HC ear drops. 


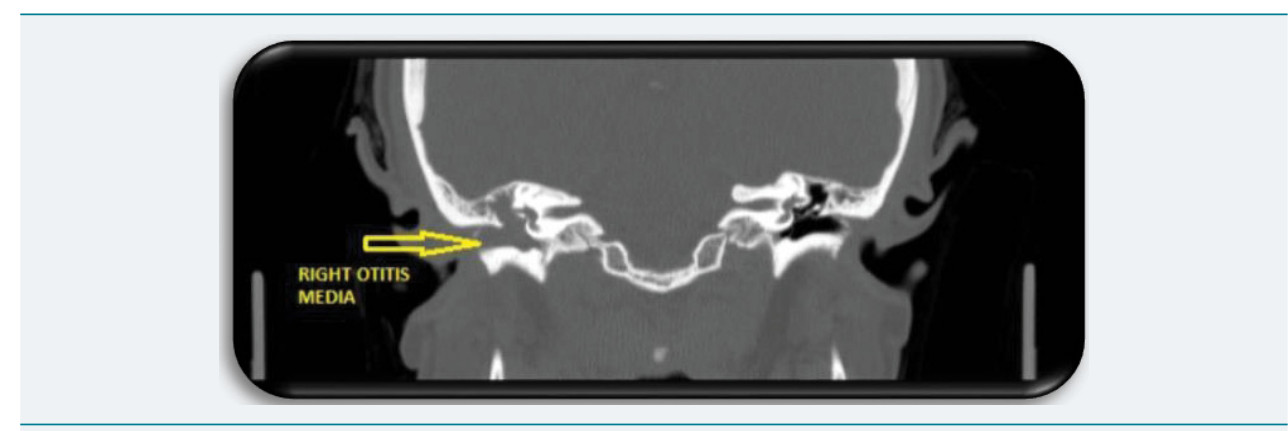

Figure 1: CT temporal bones, coronal view, demonstrating right otitis media.

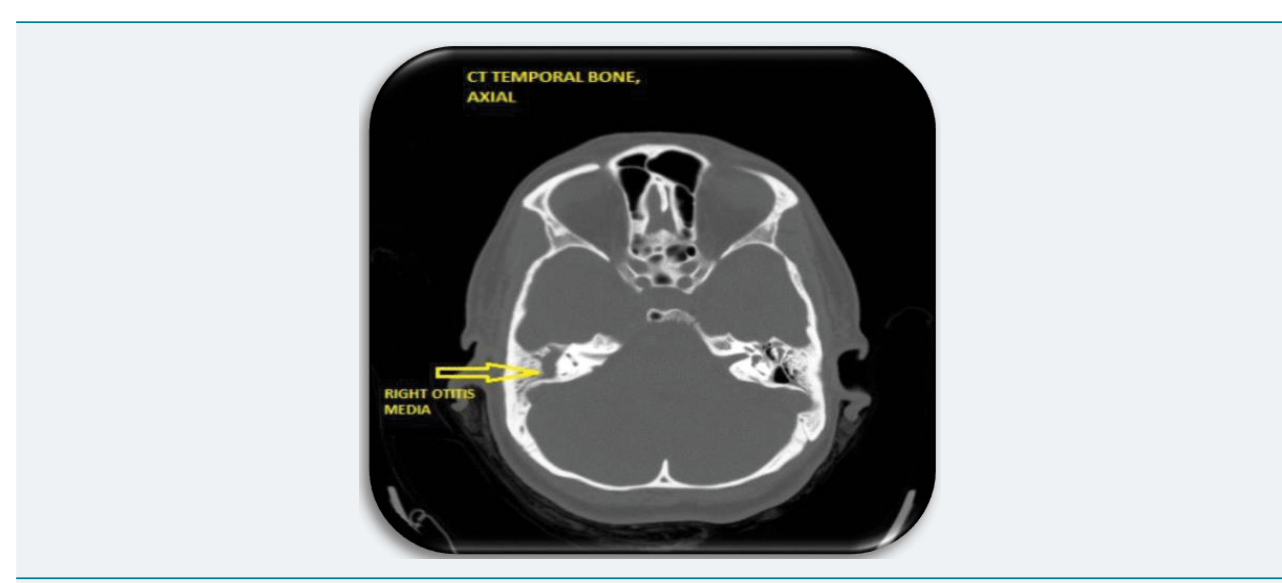

Figure 2: Axial view of CT Temporal Bone, demonstrating Right Otitis Media

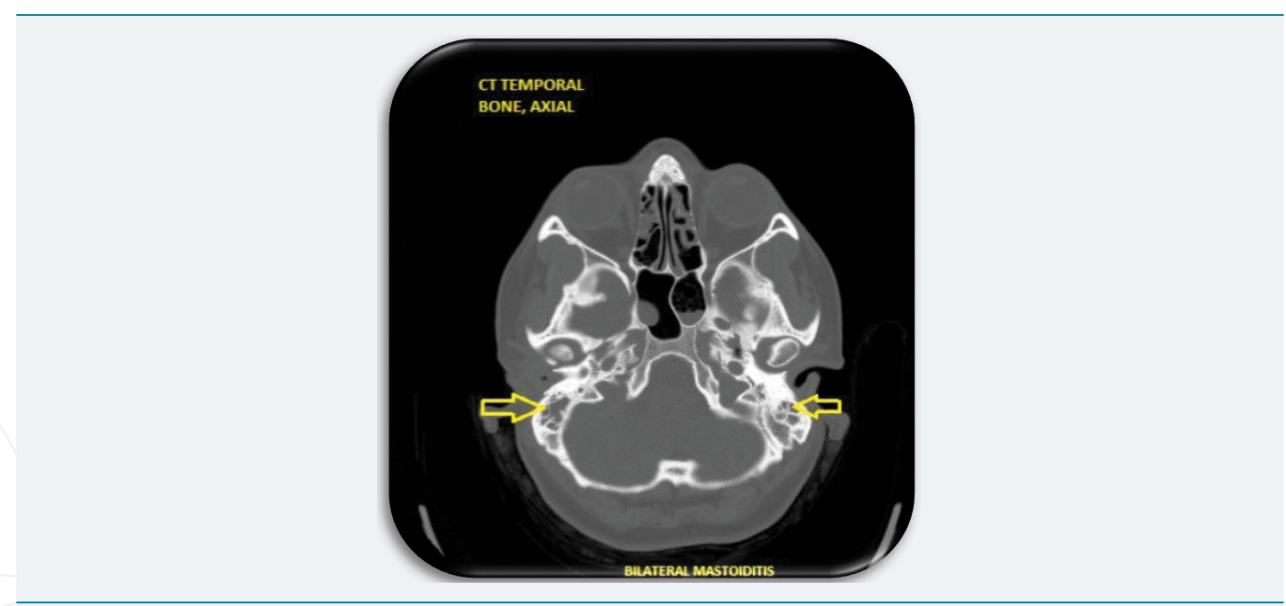

Figure 3: CT temporal bone, axial view, demonstrating bilateral mastoiditis.

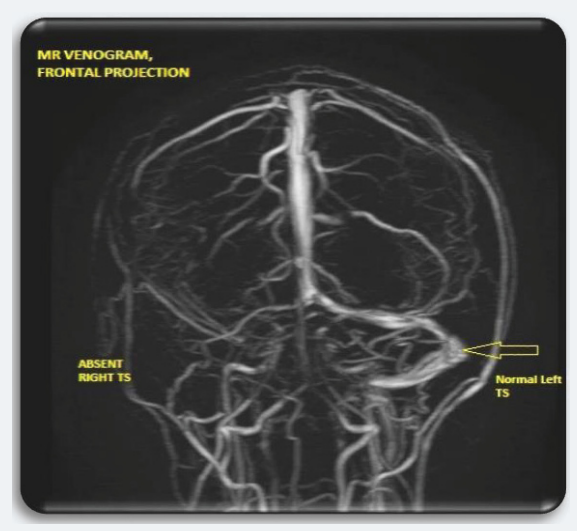

Figure 4: MR Venogram, coronal view, absent right transverse sinus. 


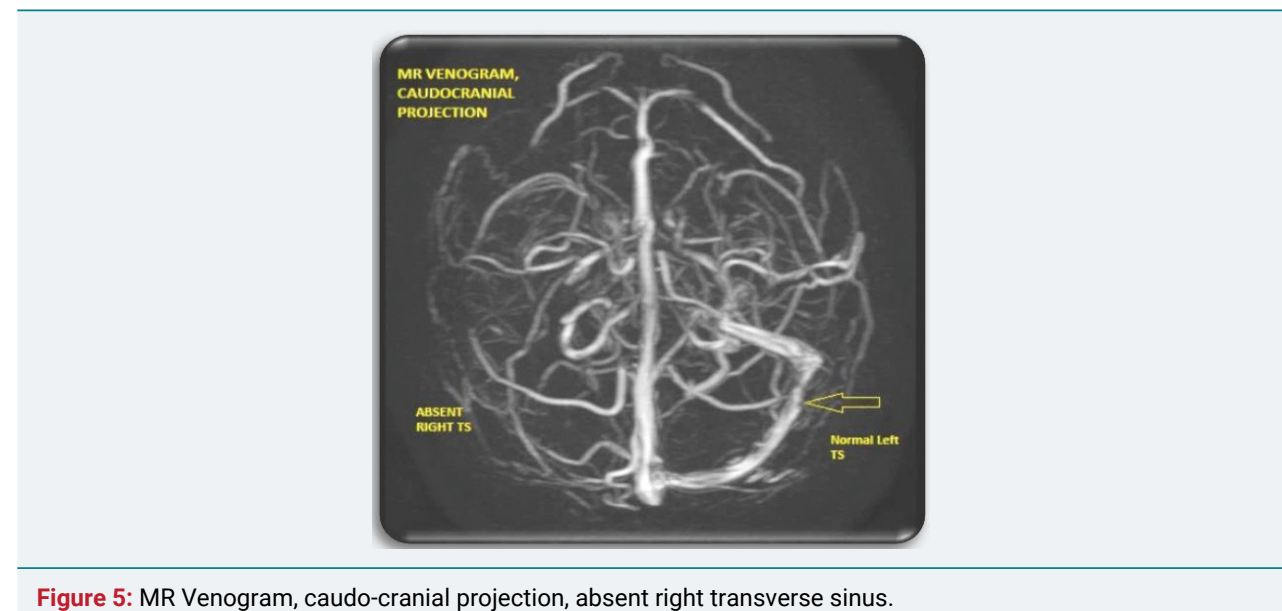

Figure 5: MR Venogram, caudo-cranial projection, absent right transverse sinus.

She remained haemodynamically stable and was transferred to a tertiary centre for neurosurgical input. The patient underwent right ear and mastoid exploration. There was no frank pus but there was some granulation tissue in the mastoid, which was removed. She was subsequently commenced on warfarin and was discharged with ENT follow up appointment after one-week and Neurology follow-up after 2 months.

\section{Management Algorithm for suspected LST in Adult (Figure 6)}

Ear examination is essential in a patient with suspected LST, as otoscopy is often the key examination that implies the patient's complaints emerged from the ears, especially if the neurological symptoms are more dominant as in this case [5]. Typical management of LST includes empirical broad-spectrum antibiotics-often a third generation cephalosporin and metronidazole-combined with mastoidectomy to remove granulation tissue adjacent to the affected sinus if the patient remains unresponsive with antibiotics alone [1,3,5,6]. Mastoidectomy should reflect the extent of the disease and may range from simple to radical mastoidectomy with additional procedures if clinically indicated [1]. There is similarly no consensus on the total duration of antibiotics [1]. Literature suggests that morbidity with early surgery may be less than that with prolonged medical management, [2] which may be a learning point from this case.

Anticoagulants are recommended by some authors to prevent the extension of the sinus thrombus, finely balanced against the risks of releasing septic emboli, drug interactions, induced thrombocytopenia and post-operative bleeding [5]. The role of anticoagulants in septic otogenic LST remains unclear, as with internal jugular vein ligation, its use does not seem to be essential for the management of LST, so should be considered, according to the extent of disease and state of patients $[3,4]$.

\section{Discussion}

MRI venography is considered the Gold Standard imaging modality for demonstrating otogenic LST. MRV is a non-invasive, highly definitive means of evaluating the presence of blood within the sinus lumen that is favourably radiation sparing, [3,6] with near 100\% sensitivity [1] MR Angiography in the venous phase is no longer advised due to the risk of embolic stroke.

Contrast enhanced Computer Tomography serves as a cost-effective screening tool for patients with suspected LST disease Au et al found that CT with contrast suggestive of LST had a sensitivity of $87 \%$ of 46 surgically confirmed cases [1]. CT demonstrates abnormalities of the mastoid and cranial cavity as seen from our patient's neuroimaging [5]. CT is also useful in demonstrating sinus wall and Delta Sign, a filling defect of the lateral sinus seen as a darkened area in the vessel lumen that signifies thrombus, within contrast-enhanced sinus wall $[1,6]$. 


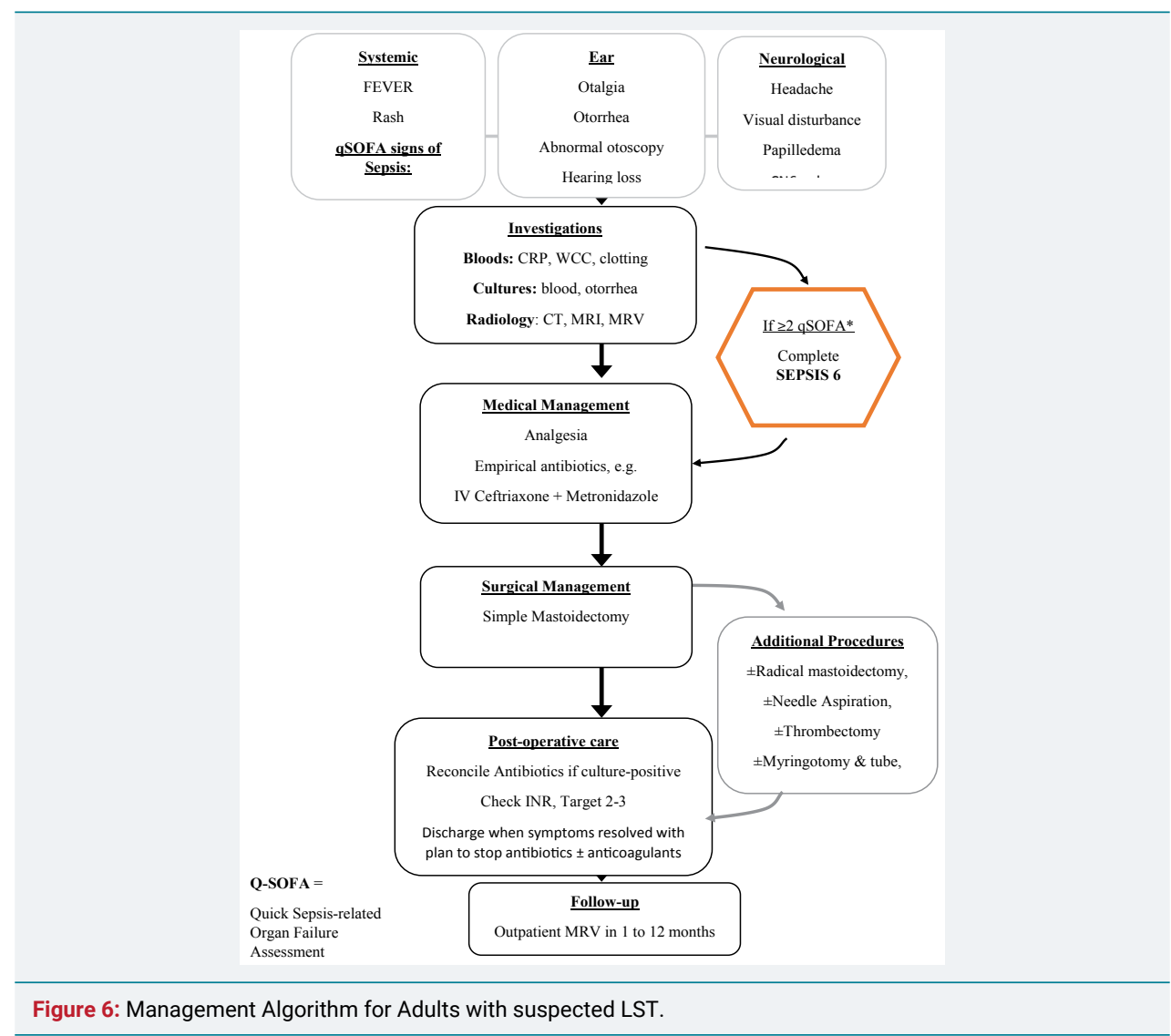

The bacteria causing otogenic LST has recently been noted to shift from the classic S. pneumonia and Beta-Haemolytic Streptococcus to mixed flora, more often finding Pseudomonas, Staphylococcus, Proteus mirabilis, methycillin-resistant Staphylococcus, Haemophilus influenza and anaerobes [5]. The change in microbiology has also been attributed in part to chronic ear disease more commonly proceeding LST than acute ear infections, as well as to the heavy pattern of antibiotic usage $[1,3,5]$. Au et al report only $46 \%$ patients had positive bacterial cultures (from blood, middle ear, discharge and abscess drainage), with a majority of cultures reporting no growth [1]. Increasing occurrence of negative cultures may result from prior antibiotic exposure [3].

The impact of antibiotics on the mortality rate of otogenic LST should not be underestimated, widely preventing the complication of septic embolic. Antibiotics combined with surgical management drove the incidence of intracranial complications of otitis media down, and subsequent mortality fell by a factor of 10 [2,4]. Antibiotics have also altered how clinicians diagnose, as the once cardinal high-grade, spiking or 'picket-fence' fever has been attenuated by previous antibiotic courses, sometimes from the community or outpatient setting [1-3]. This masking effect may make early diagnosis of LST increasingly challenging and therefore requires clinicians to maintain a high index of suspicion $[1,6]$.

Once otogenic LST has been diagnosed empirical, broad-spectrum antibiotic therapy should be commenced, given the wide range of species that have been reported to be causative agents $[1,4,6]$. Ceftriaxone has broad coverage and good penetration into the central nervous system, other common regimens include Vancomycin and Metronidazole, [1] later narrowed if the bacterial cause is speciated [1,6]. Total length of antibiotic therapy required is not well defined [4]. In the context of a protracted course of medical treatment or frequent changes in antibiotic regimens, a resistant organism must be suspected [6]. Therefore combined antibiotic and surgical treatment are employed as definitive management to keep mortality low. 
For the patient in this case, the decision to intervene surgically was based on the presence of mastoid involvement and her deterioration despite medical treatment. Prompt simple mastoidectomy alongside broad-spectrum antibiotics, is accepted as definitive management for otogenic LST, $[3,6]$ and recognised to improve the prognosis of LST on a background of cholesteatoma [1]. However there are insufficient reports about the timing and extent of the surgical procedures or the management of the thrombosed sinus, which remains disputed $[1,6]$. Many authors recommend needling the sigmoid sinus to confirm diagnosis, and clot removal to obtain blood flow in the surgery, whilst others argue that this does not improve prognosis $[3,5,6]$. Myringotomy and tube insertion alone in select cases of LST has been successful as a more conservative alternative [1]. Internal jugular vein (IJV) ligation has not been recommended for routine use since the introduction of antibiotics made the complication of septic emboli rare [3]. IJV ligation is principally reserved for refractory septicaemia, septic pulmonary emboli or LST without any organised thrombi in the mastoid area under exploration of lateral sinus, and even then approached with caution $[1,3,6]$.

Anticoagulant use is described with controversy in the literature $[3,6]$. They are reportedly efficacious when there's significant thrombus progression, neurological changes, persistent fever or an embolic event [1]. However some authors suggest that anticoagulants may cause more harm than good, with the reported risk of releasing septic emboli, [3] and postoperative haemorrhage or mastoid haematoma observed in a minority $[1,3]$.

The rationale for low-dose anticoagulants, as with our patient on LMWH and later warfarin, is prevention of thrombi formation, breakdown of thrombi extending into the IJV and transverse sinus [6] and in LST secondary to acute otitis media, which more easily disseminates beyond a localised area [3]. Intriguingly, $\mathrm{Au}$ et al found a higher rate of partial or complete sinus re-canalisation in cases where anticoagulants were used [1]. Unfortunately the roles of repeat imaging and the significance of sinus recanalization or persisting thrombosis are poorly defined, and should remain a focus for research. There is $10 \%$ morbidity at discharge observed in the otogenic LST population, mostly papilledema, cranial nerve palsy, sensorineural hearing loss, stroke and septic hip joint [1]. Earlier diagnosis, simple surgical management and the addition of antibiotics reduced the mortality rate of LST to $<10 \%[1,6]$.

\section{Conclusion}

This case report describes Lateral Sinus Thrombosis secondary to chronic supportive otitis media in a 39-year-old woman who presented with headache, fever and mastoid tenderness. Otogenic LST is scarcely reported in developed countries in recent years but continues to occur, and may have life-threatening consequences [4-6]. Contemporary literature warns us that the spiking fever and positive cultures may not be apparent due to prior use of antibiotics, so should not be relied upon for diagnosis.

We encourage investigation with CT with contrast as a screening tool, and MRI with Venography for sensitivity. Medical management is broad-spectrum intravenous antibiotics, narrowed according to Hospital Trust guidelines or positive microbiology results. The imaging and evolution of the clinical picture with medical treatment should determine the extent of mastoid surgery. The importance and timing of recanalization after LST treatment would inform patient follow-up however it remains to be widely studied in the literature [6].

Our patient was initially treated conservatively with antibiotics and anticoagulants. However as she was still symptomatic on readmission, she had a mastoid exploration. As in our case LST management currently would be clearance of granulation tissue and no sigmoid sinus surgery. Additional procedures may be required. 
As medicine advances it allows formerly brilliant investigations, procedures and treatments to retire. Antibiotics, anticoagulants, MR Venography and surgical techniques have all developed and changed over the last 150 years and this has gentrified the process of diagnosing and treating otogenic LST.

\section{References}

1. $\mathrm{Au} \mathrm{JK}$, Adam SI, Michaelides EM. Contemporary management of pediatric lateral sinus thrombosis: a twenty year review. Am J Otolaryngol. 2013; 34: 145-150. Ref.: https://goo.gl/TUPPjj

2. Goldenberg RA. Lateral sinus thrombosis: Medical or surgical treatment? Arch Otolaryngol. 1985; 111: 56-58. Ref.: https://goo.gl/FBy7M5

3. Lee JH, Choi SJ, Park K, Choung YH. Managements for lateral sinus thrombosis: does it need the ligation of internal jugular vein or anticoagulants? Eur Arch Otorhinolaryngol. 2009; 266: 51-58. Ref.: https://goo.gl/jYFbYa

4. Christensen N, Wayman J, Spencer J. Lateral sinus thrombosis: a review of seven cases and proposal of a management algorithm. Int j pedia otorhinolaryngol. 2009; 73: 581-584. Ref.: https://goo.gl/zovEQk

5. Ropposch T, Nemetz U, Braun EM, Lackner A, Tomazic PV, et al. Management of otogenic sigmoid sinus thrombosis. Otol Neurotol. 2011; 32: 1120-1123. Ref.: https://goo.gl/Jem88B

6. Iseri M, Aydn Ö, Üstündag E, Keskin G, Almaç A. Management of lateral sinus thrombosis in chronic otitis media. Otol Neurotol. 2006; 27: 1098-1103. Ref.: https://goo.gl/7bk4ar 